Palavras chave: Fixação de carbono Análise de agrupamento Grupos sucessionais

Histórico: Recebido 14/03/2012 Aceito 29/04/2014

Keywords: Carbon sink Cluster analysis Successional groups

Correspondência: luciano.watzlawick@pq.cnpq.br

DOI: | $0.1590 / 0$ | 04776020|42004|492
Luciano Farinha Watzlawick', Patrikk John Martins², Aurélio Lourenço Rodrigues ${ }^{3}$, Ângelo Augusto Ebling ${ }^{3}$, Rafaelo Balbinot ${ }^{4}$, Sebastião Brasil Campos Lustosa'

\section{TEORES DE CARBONO EM ESPÉCIES DA FLORESTA OMBRÓFILA MISTA E EFEITO DO GRUPO ECOLÓGICO}

RESUMO: O presente trabalho foi realizado no município de Boa Ventura do São Roque, $\mathrm{PR}$, com o objetivo de avaliar os teores de carbono nos compartimentos das árvores de 12 espécies arbóreas da Floresta Ombrófila Mista Montana; testar a eficácia do fator de conversão de 0,5 na estimativa do carbono nas espécies, e avaliar a influência do grupo ecológico das espécies quanto aos teores de carbono. Teores de carbono médio foram obtidos, a partir dos compartimentos das árvores e comparados entre si, entre as espécies e com o fator de conversão, visando a avaliar as diferenças interespecíficas e de eficácia do fator. Para analisar a influência dos grupos ecológicos sobre os teores de carbono, procedeu-se com a análise de agrupamentos. Não foi observada diferença significativa entre os teores de carbono encontrados para os diferentes compartimentos das árvores. Entretanto, considerando os teores das 12 espécies avaliadas, Luehea divaricata, Albizia polycephala e Cestrum sp. foram, estatisticamente, diferentes das demais, apresentando concentrações menores. Constatou-se que o fator de conversão de 0,5 superestima os teores de carbono nas árvores a uma média de $14,27 \%$. Não houve correlação entre o grupo ecológico das espécies e seus teores de carbono, considerando que houve a formação de agrupamentos por semelhança nos teores, representados por espécies de diversas fases sucessionais.

\section{CARBON CONCENTRATION IN SPECIES OF THE ARAUCARIA FOREST AND EFFECT OF THE ECOLOGICAL GROUP}

ABSTRACT: This study was conducted in Boa Ventura do São Roque, Paraná State, Brazil, aiming to evaluate the carbon concentration in components of 12 arboreal species from the Araucaria Forest, as well as the performance of the 0.5 conversion factor and the influence of trees ecological groups in their carbon concentration. Carbon concentration averages were obtained from the tree components, and compared among them, among the species and the conversion factor, to assess the interspecific differences and the reliability of the conversion factor. To analyze the influence of ecological groups over the carbon concentration of the species, cluster analyzes were performed. It was not found significant difference among the carbon concentration in the components of the trees. However, comparing the 12 species, Luehea divaricata, Albizia polycephala and Cestrum sp. differed significantly, presenting lower carbon concentration. Comparison between carbon concentration average of the species and the 0.5 conversion factor indicated that the latter overestimates the carbon concentration in the trees at an average rate of $14.27 \%$. No correlation was found between the ecological groups of the species and their carbon concentration, since groups were formed by species with distinct ecological traits.

\footnotetext{
' Universidade Estadual do Centro Oeste - Guarapuava, Paraná, Brasil

2 Faculdade Campo Real - Guarapuava, Paraná, Brasil

${ }^{3}$ Universidade Federal do Paraná - Curitiba, Paraná, Brasil

${ }^{4}$ Universidade Federal de Santa Maria - Frederico Westphalen, Rio Grande do Sul, Brasil
} 


\section{INTRODUÇÃO}

O balanço global de $\mathrm{CO}_{2}$, importante gás do efeito estufa, é fortemente influenciado pelas florestas, considerando que estas armazenam o carbono na biomassa das árvores, no sub-bosque, na serapilheira e no solo. Diferentes fitofisionomias possuem capacidades distintas de armazenar o carbono, em função dos diferentes estágios de sucessão, idade, regime de manejo, composição florística e concentração nos compartimentos das árvores das diferentes espécies (WATZLAWICK et al., 2004).

O conhecimento preciso da concentração de carbono na fitomassa arbórea é essencial para a conversão das estimativas da biomassa florestal em estoques de carbono nesses ecossistemas. Nesse sentido, a quantificação da concentração de carbono em uma série de espécies é mandatória para se compreender o potencial das florestas em capturar e acumular esse elemento (LAMLOM; SAVIDGE, 2003; MARTIN; THOMAS, 20II).

Em razão de sua praticidade, o fator de conversão de 0,5 tem sido amplamente empregado na estimativa das concentrações e estoque de carbono na biomassa acima do solo em florestas tropicais (CHAVE et al., 2008; LEWIS et al., 2009; PYLE et al., 2008; SAATCHI et al., 20II), em florestas temperadas (FANG et al., 200I; KAUPPI et al., 1995; KURZ et al., 2009), sistemas agroflorestais (SOTO-PINTO et al., 2010) e povoamentos florestais (BEETS et al., 20 I I; REDONDOBRENES et al., 2006). Entretanto, estudos indicam que a aplicação desse fator de conversão implica em erros grosseiros na estimativa dos estoques de carbono na biomassa florestal (MARTIN; THOMAS, 20II; MELSON et al., 20I I; SANER et al., 2012; THOMAS; MALCZEWSKI, 2007), pondo em risco a credibilidade dos resultados gerados por meio desse artifício. Assumese, nesse caso, que a concentração de carbono é uma função direta e constante da biomassa, desconsiderando as variações inter e intra-específicas nos processos de alocação do carbono.

A alocação de carbono nas plantas, por sua vez, decorre de uma série de condições ambientais e de características morfofisiológicas intrínsecas, podendo variar conforme o sítio e espécie. Plantas com características ecofisiológicas semelhantes são, usualmente, incluídas em um mesmo grupo ecológico, dos quais se espera, internamente, um comportamento similar no processo de alocação de carbono (CORNELISSEN et al., 2003; WRIGHT et al., 2006).
Objetivou-se, nesse trabalho, avaliar a variação na concentração de carbono entre espécies arbóreas da Floresta Ombrófila Mista e entre seus compartimentos, e comparar os valores de carbono obtidos pelo método direto com $\circ$ uso do fator de conversão de 0,5. Adicionalmente, avaliou-se a concentração de carbono, conforme o grupo ecológico das espécies.

\section{MATERIAL E MÉTODOS}

A área de estudo está localizada no município de Boa Ventura de São Roque - PR. A vegetação local compreende a Floresta Ombrófila Mista Montana, caracterizada pelo predomínio da Araucaria angustifolia (Bertol.) Kuntze, Ocotea pulchella (Nees) Mez e Ilex paraguariensis A. St.-Hil., acompanhadas das espécies Cryptocarya aschersonia Mez. e Nectandra megapotamica Nees (INSTITUTO BRASILEIRO DE GEOGRAFIA E ESTATÍSTICA - IBGE, 1992).

Os solos da região são classificados predominantemente como Latossolos e Neossolos (EMPRESABRASILEIRADEPESQUISAAGROPECUÁRIA - EMBRAPA, 2006). De acordo com a classificação de Köppen, o clima é subtropical úmido mesotérmico-Cfb, sem estação seca definida e com verão temperado.

As árvores utilizadas no estudo foram selecionadas, a partir de uma parcela permanente de $50 \mathrm{x}$ $100 \mathrm{~m}$, subdividida em 50 unidades de $10 \times 10 \mathrm{~m}$. Todos os indivíduos com Diâmetro à Altura do Peito (DAP), maior ou igual a $5 \mathrm{~cm}$, foram considerados na amostragem, e as árvores identificadas conforme o sistema APG III (ANGIOSPERM PHYLOGENY GROUP - APG, 2009).

Com base nos dados desse levantamento foram selecionadas as árvores com área basal média (dg) de cada espécie, desde que houvesse mais de três indivíduos da mesma espécie na área de estudo. Foram abatidas 26 árvores de espécies distintas para a amostragem do carbono, entretanto, apenas 12 contaram com todos os compartimentos da parte aérea, sendo essas utilizadas no presente estudo. Para a determinação da biomassa, empregou-se o método direto, por meio da simples separação dos compartimentos, conforme Sanquetta (2002). Após o corte de cada árvore, realizou-se a cubagem, conforme o método de Smalian, obtendo-se o DAP e a altura de cada indivíduo abatido. Após a cubagem, as árvores foram separadas nos compartimentos: madeira do fuste, casca do fuste, galhos vivos, galhos mortos e folhagem.

Para a determinação do peso seco, foram retiradas amostras do fuste e da casca, a partir de discos de aproximadamente $2 \mathrm{~cm}$ de espessura, nas alturas $0 \%$; 
$50 \%$ e $100 \%$. O compartimento galhos vivos foi obtido por meio do total de galhos vivos que foram desramados do fuste sem diferenciação por diâmetro e sem as folhas. O compartimento folhagem foi obtido por meio do peso total de folhas existentes na árvore, tiradas dos galhos, e o total de galhos mortos existentes na árvore compôs - compartimento galhos mortos, sem diferenciação por diâmetro. Retirou-se de cada compartimento uma amostra de $500 \mathrm{~g}$ para a determinação do peso seco.

As amostras dos compartimentos foram secas em estufas de circulação de ar, a uma temperatura de $75^{\circ} \mathrm{C}$ até atingir $\circ$ peso constante, para posterior moagem. A determinação dos teores de carbono orgânico na biomassa florestal foi efetuada pelo método Walkley-Black com calor externo, proposto por Tedesco et al. (1995). Esse método consiste na adição de $\mathrm{K}_{2} \mathrm{Cr}_{2} \mathrm{O}_{2}$ e $\mathrm{H}_{2} \mathrm{SO}_{4}$ concentrado em amostras de biomassa moída que podem variar de 0,5 a I,0 g. As amostras são agitadas e aquecidas a $150^{\circ} \mathrm{C}$ por 30 minutos. Após 0 resfriamento, adiciona-se água às amostras para cessar a reação. A leitura dos teores de $C$ é feita, então, em Espectofotômetro de Absorção Molecular.

Para avaliar as possíveis diferenças entre os teores de carbono dos compartimentos e entre as espécies, verificou-se a homogeneidade das variâncias pelo teste de Bartlett, e procedeu-se com Análise de Variância (ANOVA), considerando-se um delineamento inteiramente casualizado, realizada com o auxílio do software Assistat 7.6. As médias foram comparadas pelo teste de Scott-Knott a 95\% de probabilidade de confiança.
A superestimativa/subestimativa obtida com a utilização do fator de conversão de 0,5 , amplamente usado, foi calculada pela equação I, sendo SP\% = Superestimativa relativa; $\mathrm{C}=$ Conteúdo de carbono $(\mathrm{kg})$ presente em $1000 \mathrm{~kg}$ de biomassa.

$\mathrm{SP}=\frac{[(1000 \cdot 50)-\mathrm{C}]}{\mathrm{C}}$

As espécies foram inicialmente agrupadas em pioneiras, secundárias iniciais e secundárias tardias, de acordo com diversos autores (BACKES; IRGANG, 2002; LONGHI, 1995; LORENZI, 2002; PAULA et al., 2004; VACCARO et al., 1999). Posteriormente, agruparam-se as espécies pela análise de agrupamentos (cluster) em função dos seus teores de carbono e distância euclidiana como medida de dissimilaridade. A análise foi realizada por meio do software Statgraphics XV.II (STATGRAPHICS $X V, 2005)$, utilizando-se o método de ligação completa.

\section{RESULTADOS E DISCUSSÃO}

Os resultados dos teores médios de carbono das espécies amostradas estão relacionados na Tabela I. Os maiores teores foram observados nas folhas de Prunus brasiliensis (46,67\%), de Cupania vernalis $(45,6 \%)$ e de Nectandra lanceolata (45,54\%). Por outro lado, os menores teores foram observados nas folhas de Cestrum sp. $(36,16 \%)$ e casca do fuste de Albizia polycephala $(39,4 \%)$ e de Luehea divaricata $(40,5 \%)$.

TABELA I Teores de carbono (\%) por compartimento das espécies amostradas em fragmento de Floresta Ombrófila Mista Montana, em Boa Ventura do São Roque - PR.

TABLE I Carbon concentration (\%) in tree components of sampled species from a Montane Araucaria Forest fragment, in Boa Ventura do São Roque - PR.

\begin{tabular}{|c|c|c|c|c|c|c|c|}
\hline Espécies & CF & GV & GM & MF & Folha & Média & CV \% \\
\hline Nectandra lanceolata Nees & 44,40 & 44,77 & 45,25 & 45,36 & 45,54 & 45,06 & $\mathrm{I}, 03$ \\
\hline Vitex megapotamica (Spreng.) Moldanke & 45,39 & 44,00 & 44,95 & 45,16 & 44,83 & 44,86 & $\mathrm{I}, \mathrm{I} 7$ \\
\hline Prunus brasiliensis (Cham. \& Schltdl.) D. Dietr. & 43,43 & 45,33 & 45,16 & 43,25 & 46,67 & 44,76 & 3,19 \\
\hline Zanthoxylum rhoifolium Lam. & 44,40 & 44,78 & 44,82 & 45,36 & 43,28 & 44,52 & $\mathrm{I}, 74$ \\
\hline Matayba elaeagnoides Radlk. & 44,02 & 43,99 & 42,94 & 45,12 & 45,27 & 44,26 & 2,15 \\
\hline Nectandra megapotamica (Spreng.) Mez & 42,82 & 45,13 & 41,71 & 45,21 & 45,23 & 44,02 & 3,74 \\
\hline Cupania vernalis Cambess. & 43,43 & 43,25 & 42,87 & 44,39 & 45,60 & 43,90 & 2,50 \\
\hline Parapiptadenia rigida (Benth.) Brenan & 43,17 & 43,66 & 43,04 & 44,22 & 44,12 & 43,64 & 1,22 \\
\hline Allophylus edulis (A. St.-Hil. et al.) Hieron. ex Niederl & 42,42 & 43,09 & 43,55 & 44,77 & 43,50 & 43,46 & $\mathrm{I}, 97$ \\
\hline Luehea divaricata Mart. \& Zucc. & 40,50 & 43,35 & 42,97 & 44,34 & 42,48 & 42,72 & 3,32 \\
\hline Albizia polycephala (Benth.) Killip ex Record & 39,40 & 42,59 & 41,95 & 44,74 & 42,88 & 42,31 & 4,56 \\
\hline Cestrum sp. L. & 41,28 & 43,39 & 44,40 & 43,66 & 36,16 & 41,77 & 8,01 \\
\hline
\end{tabular}

$\mathrm{CF}=$ Casca do fuste; GV = Galho Vivo; GM = Galho Morto; MF = Madeira do Fuste; CV\% = Coeficiente de Variação. Médias comparadas pela Análise de Variância com $95 \%$ de probabilidade de confiança. 
Não houve diferenças significativas entre os teores de carbono nos diferentes compartimentos da biomassa, segundo a Análise de Variância com 5\% de nível de significância. Embora os teores de carbono dos compartimentos sejam estatisticamente iguais, o compartimento que apresentou o maior valor numérico foi a madeira do fuste $(44,63 \%)$, seguido por galhos vivos $(43,94 \%)$, folhas $(43,80 \%)$, galhos mortos $(43,63 \%)$ e casca do fuste $(42,89 \%)$.

Estudando os teores de Carbono na Floresta Ombrófila Mista, Watzlawick et al. (2004) encontraram os maiores teores de carbono nas folhas de Prunus brasiliensis (47,34\%); galhos vivos da Sloanea lasiocoma (43,69\%); galhos mortos da Nectandra megapotamica (45,23\%); casca da Ocotea pulchella (43,24\%) e madeira da Araucaria angustifolia (44, I2\%). Os mesmos autores encontraram os menores teores nas folhas de Sebastiania commersoniana (36,23\%); galhos vivos de Ocotea porosa (37,28\%); galhos mortos de Ocotea pulchella (36,24\%); casca de Cupania vernalis (34,05\%); e na madeira de Dicksonia sellowiana (39,01\%). Arias et al. (20II), avaliando teores de carbono em espécies florestais da Costa Rica, observaram maior concentração de carbono na madeira do fuste, seguido dos galhos, folhas e casca. Em outros estudos envolvendo os teores de carbono em Acacia mearnsii De Wild., no Rio Grande do Sul, Saidelles et al. (2009) verificaram diferença significativa nos teores das folhas, sendo que os demais compartimentos não apresentaram diferença significativa. Tais resultados indicam que a tendência de alocação de $C$ nos compartimentos é bastante variável conforme as espécies avaliadas.

De acordo com Ericsson et al. (1996), a dificuldade em se determinar a tendência de alocação do carbono entre as espécies e partes de uma mesma planta, deve-se: à disponibilidade de nutrientes, água, luz, $\mathrm{CO}_{2}$; idade da planta, e estágio de desenvolvimento do seu sistema radicular. No entanto, segundo o mesmo autor, existem características mais determinantes no processo de assimilação e alocação do carbono como a constituição genética da planta. Portanto, o resultado exato da interação de todos esses fatores no crescimento e distribuição do carbono é difícil de ser predito. Essas interações geram, portanto, as diferenças na alocação do carbono nos diferentes compartimentos, como observado no presente trabalho e nos estudos comparados.

Observa-se que o uso do fator de conversão de 0,5 gerou superestimativas nas concentrações de carbono para todas as espécies avaliadas (Tabela 2). As superestimativas variaram entre 10,96\% para Nectandra lanceolata, que apresentou os maiores teores médios, e 19,68\% para Cestrum sp., que apresentou os menores teores médios, ou seja, quanto menor o teor de carbono da espécie, maior o erro associado à estimativa, usandose o fator de conversão de 0,5.

TABELA 2 Média dos teores de carbono de espécies arbóreas de um fragmento de Floresta Ombrófila Mista Montana, em Boa Ventura do São Roque, PR, e superestimativas geradas com o uso do fator de conversão de 0,5 .

TABLE 2 Average carbon concentration of arboreal species from a Montane Araucaria Forest fragment, in Boa Ventura do São Roque, PR, and overestimates resulted from the use of the 0.5 conversion factor.

\begin{tabular}{lccc}
\hline Espécies & Média (\%) & $\begin{array}{c}\text { Superestimativa } \\
(\%)\end{array}$ \\
\hline Nectandra lanceolata & 45,06 & a* & 10,96 \\
Vitex megapotamica & 44,86 & a & 11,46 \\
Prunus brasiliensis & 44,76 & a & 11,71 \\
Zanthoxylum rhoifolium & 44,53 & a & 12,29 \\
Matayba elaeagnoides & 44,27 & a & 12,95 \\
Nectandra megapotamica & 44,02 & a & 13,58 \\
Cupania vernalis & 43,91 & a & 13,87 \\
Parapiptadenia rigida & 43,64 & a & 14,57 \\
Allophylus edulis & 43,47 & a & 15,03 \\
Luehea divaricata & 42,73 & b & 17,02 \\
Albizia polycephala & 42,31 & b & 18,17 \\
Cestrum sp. & 41,78 & b & 19,68 \\
\hline
\end{tabular}

* Médias seguidas da mesma letra não diferem estatisticamente de acordo com o teste de Scott-Knott, com 5\% de nível de significância.

Semelhantes aos presentes resultados, Dallagnol et al. (20I I), avaliando os teores de carbono em cinco espécies florestais concluíram que, raramente, as concentrações de carbono ultrapassam os $50 \%$. Weber et al. (2006), avaliando seis espécies da Floresta Ombrófila Mista, observaram que as concentrações de carbono variaram de $39,3 \%$ a $44,2 \%$, valores também inferiores aos $50 \%$. Outros estudos, como os desenvolvidos por Bert e Danjon (2006) e Laiho e Laine (1997), avaliando os teores de carbono em coníferas, constataram diferenças significativas entre o fator de conversão e os teores observados, gerando subestimativas nos teores entre $-5,8 \%$ e $-6,7 \%$.

São poucas as referências na literatura que constatam o oposto, como Zhang et al. (2009) que, ao estudarem 10 diferentes espécies na China, entre coníferas e folhosas, não encontraram diferença significativa entre a média dos teores das espécies e o 
fator de conversão 0,5 , embora os teores de carbono dos fustes tenham variado, significativamente, de $43,4 \%$ para a espécie Populus davidiana Dode a $55,6 \%$ para a espécie Phellodendron amurense Rupr.

No caso das espécies avaliadas neste estudo, constata-se, portanto, que a aplicação do fator de conversão é inadequada, considerando que a sua utilização resultou em superestimativa média de $14,27 \%$ nos teores de carbono das espécies avaliadas. Tais resultados não refletem as condições legítimas das espécies da floresta, podendo incorrer em estimativas errôneas, especialmente quando utilizado em projetos de grande importância, como os do Mecanismo de Desenvolvimento Limpo (MDL), e outros que requeiram estimativas precisas.

Em relação à concentração de carbono nas espécies avaliadas, Luehea divaricata, Albizia polycephala e Cestrum sp. apresentaram concentrações inferiores às demais, conforme teste de Scott-Knott a $5 \%$ de significância. Porém, quando submetidas à análise de agrupamentos, as espécies foram diferenciadas em três grupos, conforme a Figura I, com a "linha fenon" traçada na metade da distância euclidiana máxima $(I, 7)$, conforme recomendação de Barouche e Saporta (1972).

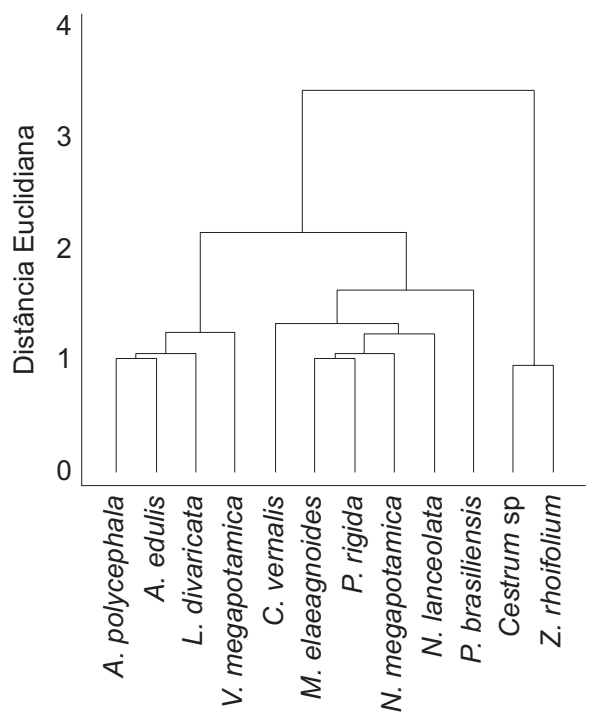

FIGURA I Agrupamento das espécies em função de seus teores de carbono em um fragmento de Floresta Ombrófila Mista Montana, em Boa Ventura do São Roque - PR

FIGURE I Species clusters according to their carbon concentration in a Montane Araucaria Forest fragment, in Boa Ventura do São Roque - PR.

As espécies resultantes do primeiro grupo foram todas classificadas como secundárias iniciais
(Luehea divaricata, Albizia polycephala, Allophylus edulis e Vitex megapotamica). O segundo grupo abrangeu cinco espécies classificadas como secundárias iniciais (Parapiptadenia rigida, Matayba elaeagnoides, Nectandra megapotamica, Cupania vernalis e Prunus brasiliensis) e uma espécie classificada como secundária tardia (Nectandra lanceolata). $\mathrm{O}$ terceiro grupo agregou as espécies Cestrum sp. e Zanthoxylum rhoifolium, classificadas como pioneira e secundária inicial, respectivamente.

Embora o primeiro agrupamento tenha sido formado apenas por espécies consideradas secundárias iniciais, os outros grupos resultantes não foram homogêneos quanto à classificação ecológica das espécies. Além do mais, os outros grupos também apresentaram espécies consideradas secundárias iniciais, que não foram, necessariamente, unidas ao primeiro grupo onde essa classificação ecológica foi absoluta.

Avaliando a capacidade de fixação de carbono de espécies de grupos sucessionais distintos, Brun (2007) concluiu que para as frações galhos vivos e mortos e na madeira (fuste), o maior potencial de acúmulo foi apresentado pelas espécies do grupo das secundárias iniciais, enquanto que na casca do fuste ocorreu uma equivalência na alocação entre os grupos das secundárias iniciais e tardias. $\mathrm{Na}$ fração folhas, a maior quantidade do elemento foi observada nas espécies do sub-bosque, que, segundo o autor, deve-se, principalmente, à típica competição por luminosidade entre as espécies formadoras desse estrato e à elevada densidade de indivíduos por área. Em relação à biomassa, Shimamoto (20/2), avaliando o acúmulo em diferentes grupos ecológicos constatou que as espécies não pioneiras acumulam quase $o$ dobro que as pioneiras.

Os resultados do presente trabalho demonstram não haver relação evidente entre os teores de carbono e o grupo ecológico das espécies, considerando a existência de indivíduos representantes de várias fases sucessionais em um único agrupamento. Tais resultados podem estar relacionados às condições ambientais favoráveis às espécies no local, sem grandes restrições, levando-as a apresentar comportamento ecofisiológico semelhante, pois, conforme constatações de Enquist et al. (1999) a não diferenciação nas concentrações de carbono entre espécies de grupos ecológicos distintos pode ser esperada em ambientes onde há suprimento constante de recursos.

Nesse caso, embora não haja variação nas concentrações de carbono relativas entre os grupos de espécie, as diferentes taxas de crescimento diamétrico e em altura usualmente observadas entre 
diferentes grupos ecológicos indicam variação nas estratégias de alocação de carbono, sendo preferível às espécies pioneiras investirem no rápido crescimento, enquanto as espécies tolerantes à sombra tendem a investir na produção de madeira de elevada densidade (CHAMBERS et al., 2004; ENQUIST et al., 1999; ENQUIST; NIKLAS, 200I).

Sugere-se, portanto, que com vistas à investigação mais aprofundada do efeito do grupo ecológico sobre as concentrações de carbono em espécies arbóreas da Floresta com Araucária, sejam agregadas outras características das espécies na análise de agrupamentos, como densidade da madeira e incremento, e, ainda, a inclusão de um número maior de espécies dos diferentes grupos ecológicos.

\section{CONCLUSÕES}

Das 12 espécies da Floresta Ombrófila Mista Montana que foram estudadas, Luehea divaricata, Albizia polycephala e Cestrum sp. mostraram diferença estatisticamente significativa quando comparadas às demais, em relação aos teores médios de carbono, apresentando valores inferiores. Os compartimentos avaliados (casca do fuste, madeira do fuste, galhos vivos, galhos mortos e folhas) não apresentaram diferenças significativas quanto ao teor de carbono. A utilização do fator de conversão de 0,5 para a determinação do teor de carbono acarretou em uma superestimativa média de 14,27\%, em relação aos valores observados, não sendo aconselhada a sua utilização. O grupo ecológico das espécies não foi determinante em seus teores de carbono, considerando que houve a formação de agrupamentos por semelhança nos teores, representados por espécies de mais de uma fase sucessional, e a ocorrência de espécies de um mesmo grupo ecológico em todos os agrupamentos.

\section{REFERÊNCIAS}

ANGIOSPERM PHYLOGENY GROUP. An update of the Angiosperm Phylogeny Group classification for the orders and families of flowering plants: APG III. Botanical Journal of the Linnean Society, London, v. 16I, n. 2, p. I05-121, 2009.

ARIAS, D.; CALVO-ALVARADO, J.; RICHTER, D. B.; DORENBUSCH, A. Productivity, aboveground biomass, nutrient uptake and carbon content in fast-growing tree plantations of native and introduced species in the Southern Region of Costa Rica. Biomass and Bioenergy, Oxford, v. 35, n. 5, p. I779-I788, 201 I.
BACKES, P.; IRGANG, B. E. Árvores do Sul: guia de identificação e interesse ecológico. Santa Cruz do Sul: Instituto Souza Cruz, 2002. 326 p.

BAROUCHE, J. M.; SAPORTA, G. Análise de dados. Rio de Janeiro: Zaar, 1972. II6 p.

BEETS, P. N.; BRANDON, A. M.; GOULDING, C. J.; KIMBERLEY, M. O.; PAUL, T. S. H.; SEARLES, N. The inventory of carbon stock in New Zealand's post-1989 planted forest for reporting under the Kyoto protocol. Forest Ecology and Management, Amsterdam, v. 262, p. III9-II30, 20II.

BERT, D.; DANJON, F. Carbon concentration variations in the roots, stem and crown of mature Pinus pinaster (Aait.). Forest Ecology and Management, Amsterdam, v. 222, p. 279-295, 2006.

BRUN, F. G. K. Dinâmica do Carbono orgânico em espécies arbóreas de uma Floresta Estacional Decidual no Rio Grande do Sul. 2007. 107 f. Dissertação (Mestrado em Engenharia Florestal) - Universidade Federal de Santa Maria, Santa Maria, 2007.

CHAMBERS, J. Q.; HIGUCHI, N.; TEIXEIRA, L. M.; SANTOS, J.; LAURANCE, S. G.; TRUMBORE, S. E. Response of tree biomass and wood litter to disturbance in a Central Amazon forest. Oecologia, Berlin, v. I4I, p. 596-6II, 2004.

CHAVE, J.; CONDIT, R.; MULLER-LANDAU, H. C.; THOMAS, S. C.; ASHTON, P. S.; BUNYAVEJCHEWIN, S.; CO, L. L.; DATTARAJA, H. S.; DAVIES, S. J.; ESUFALI, $\mathrm{S}$. Assessing evidence for a pervasive alteration in tropical tree communities. PLoS Biology, San Francisco, v. 6, p. 455-462, 2008.

CORNELISSEN, J. H. C.; LAVOREL, S.; GARNIER, E.; DIAZ, S.; BUCHMANN, N.; GURVICH, D. E.; REICH, P. B.; STEEGE, H.; MORGAN, H. D.; HEIJDEN, M. G. A.; PAUSAS, J. G.; POORTER, H. A handbook of protocols for standardised and easy measurement of plant functional traits worldwide. Australian Journal of Botany, Melbourne, v. 5I, p. 335380, 2003.

DALLAGNOL, F. S.; MOGNON, F; SANQUETTA, C. R.; DALLA CORTE, A. P. Teores de carbon em cinco espécies florestais e seus compartimentos. Floresta e Ambiente, Seropédica, v. 18, n. 4, p. 410-416, 201 I.

EMPRESA BRASILEIRA DE PESQUISA AGROPECUÁRIA. Centro Nacional e Pesquisa em Solos. Sistema brasileiro de classificação de solos. Brasília: EMBRAPA-SPI; Rio de Janeiro: EMBRAPA-Solos, 2006. 306 p.

ENQUIST, B. J.; NIKLAS, K. J. Invariant scaling relations across tree-dominated communities. Nature, London, v. 410, p. 655-660, 2001 . 
ENQUIST, B. J.; WEST, G. B.; CHARNOV, E. L.; BROWN, J. H. Allometric scaling of production and life-history variation in vascular plants. Nature, London, v. 40I, p. 907-91 I, 1999.

ERICSSON, T.; RYTTER, L.; VAPAAVOURIT, E. Physiology of carbon allocation in trees. Biomass and Bioenergy, Oxford, v. II, n. 3, p. II5-127, 1996.

FANG, J. Y.; OIKAWA, T.; KATO, T.; MO, W. H.; WANG, Z. H. Biomass carbon accumulation by Japan's forests from 1947 to 1995. Global Biogeochemical Cycles, Washington, v. 19, p. I-10, 2005.

INSTITUTO BRASILEIRO DE GEOGRAFIA E ESTATÍSTICA. Manual técnico da vegetação brasileira. Rio de Janeiro, 1992. $92 \mathrm{p}$.

KAUPPI, P. E.; TOMPPO, E.; FERM, A. C and $\mathrm{N}$ storage in living trees within Finland since 1950s. Plant and Soil, The Hague, v. 168, p. 633-638, 1995.

KURZ, W. A.; DYMOND, C. C.; WHITE, T. M.; STINSON, G.; SHAW, C. H.; RAMPLEY, G. J.; SMYTH, C.; SIMPSON, B. N.; NEILSON, E. T.; TROFYMOW, J. A.; CBM-CFS3: a model of carbon-dynamics in forestry and land-use change implementing IPCC standards. Ecological Modelling, Amsterdam, v. 220, p. 480-504, 2009.

LAIHO, R.; LAINE, J. Tree stand biomass and carbon content in an age sequence of drained pine mires in southern Finland. Forest Ecology and Management, Amsterdam, v. 93, p. I6I-169, 1997.

LAMLOM, S. H.; SAVIDGE, R. A. A reassessment of carbon content in wood: variation within and between 4I North American species. Biomass Bioenergy, Oxford, v. 25, p. 38I-388, 2003.

LEWIS, S. L.; LOPEZ-GONZALEZ, G.; SONKE, B.; AFFUMBAFFOE, K.; BAKER, T. R.; OJO, L. O.; PHILLIPS, O. L.; REITSMA, J. M.; WHITE, L.; COMISKEY, J. A. Increasing carbon storage in intact African tropical forests. Nature, London, v. 457, p. 1003-1006, 2009.

LONGHI, R. A. Árvores e arvoretas do sul. 2. ed. Porto Alegre: L\&P, 1995. 176 p.

LORENZI, H. Árvores brasileiras: manual de identificação e cultivo de plantas arbóreas nativas do Brasil. 4. ed. Nova Odessa: Instituto Plantarum, 2002. v. I, 384 p.

MARTIN, A. R.; THOMAS, S. C. A reassessment of carbon content in tropical trees. PLoS One, San Francisco, v. 6, p. I-9, 201 I.

MELSON, S. L.; HARMON, M. E.; FRIED, J. S.; DOMINGO, J. B. Estimates of live-tree carbon stores in the Pacific Northwest are sensitive to model selection. Carbon Balance Management, London, v. 6, p. I-16, $201 \mathrm{I}$.
PAULA, A. de; SILVA, A. F.; MARCO, P. de; SANTOS, F. A. M.; SOUZA, A. L. Sucessão ecológica da vegetação arbórea em uma Floresta Estacional Semidecidual. Acta Botanica Brasilica, Porto Alegre, v. 18, n. 3, p. 407-423, 2004.

PYLE, E. H.; SANTONI, G. W.; NASCIMENTO, H. E. M.; HUTYRA, L. R.; VIEIRA, S.; CURRAN, D. J.; HAREN, J. van; SALESKA, S. R.; CHOW, V. Y.; CARMAGO, P. B. Dynamics of carbon, biomass, and structure in two Amazonian forests. Journal of Geophysics Research, Malden, v. I I 3 , p. I-20, 2008.

REDONDO-BRENES, A.; MONTAGNINI, F. Growth, productivity, aboveground biomass, and carbon sequestration of pure and mixed native tree plantations in the Caribbean lowlands of Costa Rica. Forest Ecology and Management, Amsterdam, v. 232, p. I68-I78, 2006.

SAATCHI, S. S.; HARRIS, N. L.; BROWN, S.; LEFSKY, M.; MITCHARD, E. T. A.; SALAS, W.; ZUTTA, B. R.; BUERMANN, W.; LEWIS, S. L.; HAGEN, S. Benchmark map of forest carbon stocks in tropical regions across three continents. Proceedings of the National Academy of Sciences of the United States, Washington, v. 108, p. 9899-9904, 20II.

SAIDELLES, F. L. F.; CALDEIRA, M. V. W.; SCHUMACHER, M. V.; BALBINOT, R. Uso de equações para estimar carbono orgânico em plantações de Acacia mearnsii De Wild. Revista Árvore, Viçosa, v. 33, n. 5, p. 907-915, 2009.

SANER, P.; LOH, Y. Y.; ONG, R. C.; HECTOR, A. Carbon stocks and fluxes in tropical lowland Dipterocarp rainforests in Sabah, Malaysian Borneo. PLoS One, San Francisco, v. 7, p. I-II, 2012.

SANQUETTA, C. R. Métodos de determinação de biomassa florestal. In: SANQUETTA, C. R.; WATZLAWICK, L. F; BALBINOT, R.; ZILIOTO, M. A.; GOMES, F. S. (Ed.). As florestas e o carbono. Curitiba: UFPR, 2002. p. II9140.

SHIMAMOTO, C. Y. Estimativa do crescimento e acúmulo de biomassa em espécies arbóreas, como subsídio a projetos de restauração da Mata Atlântica. 20I2. 5I f. Dissertação (Mestrado em Ecologia e Conservação) Universidade Federal do Paraná, Curitiba, 2012.

SOTO-PINTO, L.; ANZUETO, M.; MENDOZA, J.; FERRER, G. J.; DE JONG, B. Carbon sequestration through agroforestry in indigenous communities of Chiapas, Mexico. Agroforest Systems, Amsterdam, v. 78, p. 395I, 2010.

STATGRAPHICS XV. Warrenton: Statistical Graphics Corporation, 2005.

TEDESCO, M. J. et al. Análises de solo, plantas e outros materiais. Porto Alegre: UFRGS, 1995. 174 p. 
THOMAS, S. C.; MALCZEWSKI, G. Wood carbon content of tree species in eastern China: interspecific variability and the importance of the volatile fraction. Journal of Environment Management, New York, v. 85, p. 659-662, 2007.

VACCARO, S.; LONGHI, S. J.; BRENA, D. A. Aspectos da composição florística e categorias sucessionais do estrato arbóreo de três subseres de uma floresta Estacional Decidual, no município de Santa Tereza, RS. Ciência Florestal, Santa Maria, v. 9, n. I, p. I-18, 1999.

WATZLAWICK, L. F. et al. Teores de carbono em espécies da Floresta Ombrófila Mista. In: SANQUETTA, C. R. et al. (Ed.). Fixação de carbono: atualidades, projetos e pesquisas. Curitiba: UFPR, 2004. p. 95-109.
WEBER, K. S.; BALBINOT, R.; WATZLAWICK, L. F.; SANQUETTA, C. R. Teores de carbono orgânico de seis espécies naturais do ecossistema da Floresta Ombrófila Mista. Ambiência, Guarapuava, v. 2, n. 2, p. 167-177, 2006.

WRIGHT, J. P.; NAEEM, S.; HECTOR, A.; LEHMAN, C.; REICH, P. B.; SCHMID, B.; TILMAN, D. Conventional functional classification schemes underestimate the relationship with ecosystem functioning. Ecology Letters, Oxford, v. 9, p. III-I20, 2006.

ZHANG, Q. et al. Carbon concentration variability of 10 Chinese temperate tree species. Forest Ecology and Management, Amsterdam, v. 258, n. 5, p. 722-727, 2009. 